\title{
ASCA observations of Galactic rotation-powered pulsars
}

\author{
M. J. Pivovaroff and V. M. Kaspi \\ MIT, Cambridge, MA 02139, USA \\ E. V. Gotthelf \\ Columbia, U., New York, NY 10027, USA
}

\begin{abstract}
We have examined several archival $A S C A$ observations of Galactic radio pulsars, including PSRs B1046-58 and B1610-50. X-ray emission is detected from PSR B1046-58 with a significance of $\sim 5 \sigma$. We find no evidence for pulsations. We argue that the emission is from a spatially unresolved synchrotron nebula powered by the pulsar. The location of the X-ray counterpart within the $95 \%$ position error ellipse of the gamma-ray source 3EG J1048-5840 strengthens the claim of Kaspi et al. (2000) that PSR B1046-58 emit $\gamma$-rays. X-ray emission from PSR B1610-50 is not detected. We use the X-ray luminosity upper limit to constrain the pulsar's velocity $\lesssim 200 \mathrm{~km} \mathrm{~s}^{-1}$, arguing against an association with the nearby supernova remnant Kes 32 . Our results for these radio pulsars contradict previous reports of detections of large (tens of arc minutes) associated synchrotron nebulae.
\end{abstract}

X-ray observations of radio pulsars provide a powerful diagnostic of the energetics and emission mechanisms of rotation-powered neutron stars, as a significant fraction of the luminosity emerges as a relativistic wind of positrons and electrons. When confined by the surrounding medium, an observable synchrotron nebula results. Measurements of the morphology and synchrotron spectrum are essential for determining the content and energy spectrum of the wind, probing the ambient density, and understanding the shock acceleration mechanism. Here, we briefly discuss the $A S C A$ observations of the young, energetic pulsars PSRs B1046-58 and B1610-50. Previous X-ray work on these pulsars had been limited (Becker \& Trümper 1997; Kawai, Tamura, \& Saito 1998 [KTS98]). We perform a detailed analysis of the ASCA data, with an emphasis on image analysis, in hopes of detecting and characterizing the $\mathrm{X}$-ray properties of these rotation-powered pulsars. For the complete analysis, see Pivovaroff, Kaspi \& Gotthelf (2000).

The left panel of Fig. 1 shows the hard $(2-10 \mathrm{keV})$ image from the SIS. We identify Src 1, which lies $20^{\prime \prime}$ from the position of the radio pulsar (Stappers, Gaensler \& Johnston 1999), as the counterpart of the pulsar. The hardness of Src 1 argues against thermal emission from the neutron star surface and supports its interpretation as non-thermal emission. Magnetospheric emission is unlikely, given the pulsed upper limit of $31 \%(3 \sigma)$ obtained from folding GIS data at the radio ephemeris. Thus, we argue that Src 1 is a spatially un resolved $\left(<1^{\prime}\right)$ synchrotron nebula powered by the pulsar. Src 1 is also the only hard 

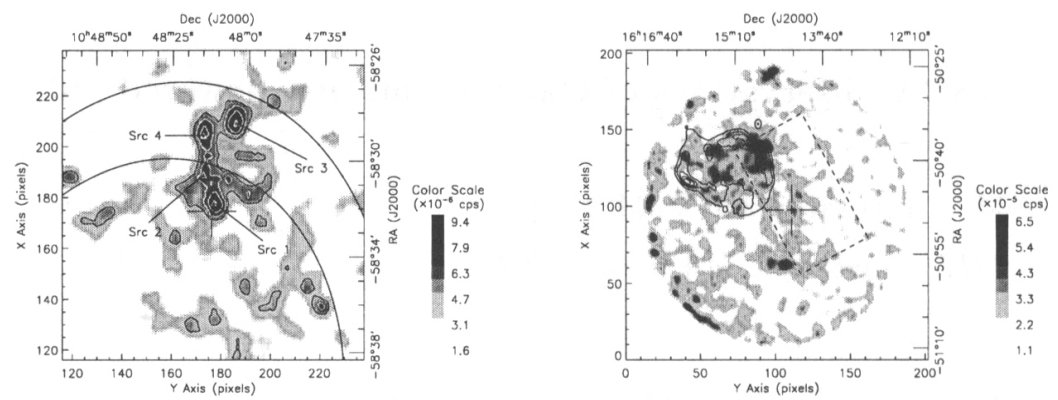

Figure 1. Left: The SIS image of PSR B1046-58. Only three of the four sources are detected in this band. The ellipses are the $95 \%$ and 99\% position contours of the EGRET source 3EG J1048-5840. Right: The GIS image of PSR B1610-50. Only emission from SNR Kes 32 is detected. The contours are from MOST $843 \mathrm{MHz}$ observations. A cross marks the pulsar location in each image.

source within the $95 \%$ error ellipse of the previously identified EGRET $\gamma$-ray source 3EG J1048-5840. Taken with the $\gamma$-ray pulsations reported by Kaspi et al. (2000), our X-ray detection of the pulsar strengthens the argument for identifying PSR B1046-58 as one of the few radio pulsars to emit $\gamma$-rays.

The right panel of Fig. 1 shows the $2-10 \mathrm{keV}$ GIS image of PSR B1610-50. No X-ray emission is detected from the pulsar. The upper limit on X-ray luminosity $L_{x}$ implies a conversion efficiency of spin-down luminosity $\dot{E}$ into X-rays less than $6 \times 10^{-4}$ in the $2-10 \mathrm{keV}$ band. Following the approach of Gotthelf \& Kaspi (1998), we use limit on $L_{x}$ to constrain the pulsar's velocity to be less than $170 \mathrm{~km} \mathrm{~s}^{-1}$. The velocity required for the association of PSR B1610-50 with SNR Kes 32 proposed by Caraveo (1993) exceeds this upper limit by a factor of 20 , arguing against the putative association.

The non-detection of X-rays from this pulsar and the spatially unresolved nebula around PSR B1046-58 contradicts previous reports (KTS98) of large $A S C A$-observed nebulae associated with these pulsars.

\section{References}

Becker, W. \& Trümper, J. 1997, A\&A, 326, 682

Caraveo, P. A. 1993, ApJ, 415, L111

Gotthelf, E. V. \& Kaspi, V. M., 1998, ApJ, 497, L29

Kawai, N., Tamura, K., \& Saito, Y. 1998, Adv. Space Res., 21, 213

Kaspi, V. M. et al. 2000, ApJ, 528

Pivovaroff, M. J., Kaspi, V. M. \& Gotthelf, E. V. 2000, ApJ, 528

Stappers, B. W., Gaensler, B. M. \& Johnston, S. 1999, MNRAS, 308, 609 